\title{
Análisis de desempeño del IEEE802.11 para la conectividad de zonas rurales de Colombia
}

\author{
Performance analysis of the IEEE802.11 for the conectivity \\ of rural zones in Colombia
}

\author{
Óscar Gualdrón González \\ Ph. D en Física, Université Laval Canada \\ Docente Tiempo Completo, Director Grupo CPS, \\ Universidad Industrial de Santander UIS \\ Bucaramanga, Colombia \\ ogualdron@uis.edu.co
}

\author{
Ricardo Andrés Díaz Suárez \\ MIE en Ingeniería electrónica, \\ Universidad Industrial de Santander \\ Docente Medio Tiempo, Investigador Grupo GITI, \\ Universidad Cooperativa de Colombia UCC \\ Bucaramanga, Colombia \\ ricardo.diazsu@campusucc.edu.co
}

\begin{abstract}
Resumen- Dentro de este artículo se presenta las características de desempeño del estándar IEEE802.11 en enlaces punto a punto de largo alcance sobre emplazamientos rurales en Colombia. Para explicar este desempeño primero se realiza una descripción detallada del comportamiento de la capa física y MAC en el despliegue de redes de largo alcance, esto se realiza mediante análisis de la regulación existente para la máxima potencia isotrópica radiada equivalente en la banda ISM, las pérdidas por propagación, el nivel de recepción de los radios Wi-Fi comerciales, la tasa de error de frame y considerando como los parámetros DIFS, Slottime y ACKTimeout que hacen parte del control de acceso al medio e inciden en la implementación de radio enlaces de varios kilómetros. Posteriormente a partir de unos modelos teóricos presentes en la literatura y uno propuesto por los autores se calcula el throughput UDP saturado unidireccional y bidireccional en función de la distancia consideradas las diferentes velocidades de transmisión; después con un par de prototipos de comunicación Wi-Fi autónomos alimentados con energía fotovoltaica diseñados y construidos en laboratorio, se realizan un grupo de medidas experimentales de throughput UDP saturado en enlaces punto-punto entre Bucaramanga y emplazamientos rurales circundantes a su área metropolitana en el rango de distancias de $\mathbf{0}-\mathbf{1 0 . 4} \mathrm{km}$, las mediciones se realizaron con el generador de tráfico IPERF enviando paquetes UDP de forma unidireccional y bidireccional, posteriormente las mediciones realizadas se comparan con los obtenidos de forma teórica.
\end{abstract}

Palabras clave - IEEE802.11, largo alcance, Física, MAC, Modelo, Throughput, Iperf.

Abstract- In this paper, we present the performance characteristics of IEEE802.11 standard in point to point reaching over rural sites in Colombia. To explain this performance is first should be carried out a detailed description of the behavior of the physical and MAC layer in the deployment of long-range networks, this is done by analyzing the existing regulation for maximum equivalent isotropic radiated power in the ISM band, the propagation losses, the reception level of commercial Wi-Fi radios, the frame error rate and considering the parameters DIFS, and ACKTimeout SLOTTIME that are part of medium access control affect the implementation of radio links of several kilometers. Following from this theoretical models in the literature and one proposed by the authors calculate the saturated throughput UDP unidirectional and bidirectional function of the distance considering the different transmission speeds; After a couple of prototype autonomous Wi-Fi communication photovoltaic powered laboratory designed and built, a group performed experimental measurements of saturated UDP throughput in point to point links between Bucaramanga and rural sites surrounding metropolitan area in the range of $0-10.4 \mathrm{~km}$ distances, measurements are performed using the iperf traffic generator sending UDP packets of unidirectional and bidirectional, then the measurements are compared with those obtained theoretically.

Keywords - IEEE802.11, long distance, MAC, Physics, Model, Throughput, iperf.

\section{INTRODUCCIÓN}

En algunas zonas rurales del mundo que hacen parte de países subdesarrollados como Colombia se carece de soluciones tecnológicas que permitan tener conectividad con el resto del mundo, como resultado estas regiones se encuentran en algunos casos marginadas y desprotegidas, lo cual permite que abunde el analfabetismo, se carezca de buenos mecanismos de salubridad pública, no exista prevención remota contra posibles desastres naturales, estos y otros factores disminuyen sustancialmente la calidad de vida y el posible desarrollo de estos emplazamientos. Los gobiernos de estos paí- 
ses tratan de mitigar esa situación al generar proyectos que permitan tener conectividad en estos emplazamientos e incentivan programas donde se ofrecen las TIC, como un mecanismo para fortalecer y gestionar las iniciativas que permitan mejorar la calidad de vida en estas comunidades. [1][2][3][4][5]

Los inconvenientes para ofrecer conectividad en zonas rurales están determinados por las limitaciones económicas, las severas condiciones ambientales, el costo de los equipos de comunicación, la carencia de infraestructura, los costos de licencia en la banda del espectro electromagnético, la carencia de un buen suministro eléctrico, el mantenimiento de los equipos y los costos que imponen los ISP (proveedores de servicio de internet) para acceder al backbone.[1][3][6]

Considerado lo anterior se necesitan tecnologías de comunicación con buenas prestaciones y de bajo costo, que permitan disminuir la brecha digital y contribuir al desarrollo de estos emplazamientos al conectarlos con el resto del mundo.

En el mercado existen diferentes tecnológicas de comunicación que permiten ofrecer conectividad en zonas rurales se encuentran: VSAT (Very Small Aperture Terminal), CDMA450 (Code Division Multiple Access), DECT (Digital Cordless Phone System), HFC (Hibrid Fiber Coaxial Networks), Redes PLC (Power Line Communications), EV-DO (Evolution-Data Optimized), GPRS (General Packet Radio Service) y Wi-Fi (Wireless Fidelity). A partir de las características de desempeño y costo algunos estudios consideran a Wi-Fi como una de las mejores alternativas para la conectividad de zonas rurales. [7][8]

Debido a la masificación en el uso de radios Wi-Fi su costo ha disminuido considerablemente, además, si se considera que estos operan en la banda ISM (Industrial, científica y médica), sus velocidades de transmisión máxima es de 11Mbps en IEEE802.11b, 54Mbps para IEEE802.11a/g y de 300Mbps para IEEE802.11n esto suponiendo canal de $40 \mathrm{MHz}$ y MIMO de $2 \times 2$. Esta tecnología permite ofrecer soluciones de conectividad de banda ancha, además, si se incorpora que al realizar variaciones en los tiempos definidos en la capa
MAC (CSMA/CA) y física definidos en el estándar o modificado el control de acceso al medio (TDMA) se puede utilizar para desplegar redes de área extensa con buenas prestaciones, estos aspectos descritos presentan a WiFi como una de las mejores opciones para ofrecer conectividad en zonas rurales. Esto ha incentivado en los últimos años varias iniciativas tanto en grupos de investigación como en empresas al desarrollo de equipos que utilizan la capa física de WiFi con modificaciones en el control de acceso al medio o con protocolos propietarios para conectar emplazamientos rurales.

En la actualidad existen algunas mediciones experimentales de throughput sobre el estándar IEEE802.11 en algunas zonas rurales de la Amazonia Peruana (1-50km) [6] y en emplazamientos rurales en Europa 10-300km [9] [10][11], además existen estudios de desempeño en redes de largo alcance considerado el emulador de canal (SR5500) para diferentes distancias en el intervalo de $(0-100 \mathrm{~km})$ [6][12] [13].

En la primera sección de este artículo se especifican algunas características del estándar IEEE802.11 el cual está diseñado y optimizado para redes de área local, en la segunda sección se especifica algunas características del desempeño de este estándar sobre una red de largo alcance que presenta un análisis de los límites que impone la capa física consideradas la PIRE, las pérdidas por propagación, nivel de señal recibida en el radio Wi-Fi y la tasa de error de frames, después se presenta el desempeño que impone la capa MAC en función de los parámetros DIFS, Slottime y ACKTimeout que hacen parte del estándar. En la tercera sección se presentan modelos teóricos para el cálculo del throughput UDP unidireccional y bidireccional sobre enlaces punto a punto IEEE802.11 de largo alcance, realizando el análisis cuando el flujo del tráfico es unidireccional y bidireccional además se propone un modelo para el cálculo del throughput basado en una máquina de estados que representa la función de coordinación distribuida en función de la distancia y se compara con el modelo propuesto por J. Simo [6]; en la cuarta sección se presenta un grupo de medidas experimentales del throughput sobre enlaces punto a punto de largo al- 
cance en zonas rurales circundantes al área metropolitana de Bucaramanga en el rango de $0-10.4 \mathrm{~km}$, estas medidas se realizaron con el generador de tráfico iperf, los nodos Wi-Fi se le configuraron los parámetros analizados en la MAC sobre el controlador del radio para mejorar el desempeño en cuanto al throughput sobre enlaces de largo alcance.

\section{CARACTERÍSTICAS DE LA CAPA FÍSICA Y MAC DEL IEEE802.11}

\section{A. Capa física IEEE 802.11}

El estándar IEEE802.11b define en su capa física la técnica de modulación de espectro ensanchado por secuencia directa de alta tasa HR/DSSS define velocidades de transmisión, 1, 2 y 5.5Mbps con modulaciones DBPSK, DQPSK, CCK respectivamente. El control de acceso del canal lo realiza a través del sensado de portadora. [14][15]En el estándar IEEE 802.11 g en su capa física define la multiplexación por división de frecuencias ortogonales OFDM para el envío de datos, la cual fracciona el canal en un número de subcanales ortogonales los cuales deben ser usados en paralelo para aumentar la transferencia de datos, utiliza un ancho de banda de $20 \mathrm{MHz}$ que se encuentra ocupado por 52 portadoras. Para la transmisión de la información, el estándar IEEE802.11g define las modulaciones 16QAM y 64QAM para 36 y 54Mbps respectivamente. El control para acceder al canal y evaluar si este está libre combina un umbral mínimo de energía con la capacidad de detectar una señal Wi-Fi válida. [14][15]

\section{B. Capa MAC IEEE802.11}

La capa MAC del estándar IEEE 802.11 define dos modos para su funcionamiento el primero llamado PCF (Point Coodination Function) y segundo DCF (Distributed Coordination Function), aunque en los radios $\mathrm{Wi}-\mathrm{Fi}$ comerciales el más implementado es el distribuido el cual será analizado a continuación.

La función de coordinación distribuida DCF utiliza el protocolo CSMA/CA (Carrier Sense Multiple Access With Collision Avoidance) para coordinar la forma en que varias estaciones acceden al canal de comunicación. Cuando una estación desea transmitir un paquete en modo DCF, primero debe activar el mecanismo CS (Carrier Sense) para determinar si hay otra estación que transmite Si encuentra el canal libre durante un intervalo de tiempo DIFS (DCF Interframe Space) o EIFS (Extended Inter Frame Space), lo cual depende si la estación estuvo involucrada en su anterior transmisión en una colisión, inicializa la etapa de contención o algoritmo backoff el cual se encuentra dividido en ranuras. El número de ranuras se selecciona de forma pseudo aleatoria de una distribución uniforme a partir del intervalo de valores. $\left[0, C W_{\min }\right]$ Cada vez que la estación transmisora considera el canal libre CS/CCA (Carrier Sense/Clear Channel Assessment), decrementa un slot. Si encuentra el canal ocupado la estación congela el algoritmo backoff hasta que encuentre el canal libre durante un DIFS. Cuando el número de ranuras Ilega a cero la estación comienza a transmitir. Al terminar la transmisión la estación transmisora espera un ACK que será enviado desde la estación receptora en el caso que no ocurra el arribo de un ACK durante un intervalo de tiempo ACKTimeout se considera que existió una colisión (las estaciones no logran diferenciar una colisión de una pérdida de paquete). La estación transmisora dobla la ventana de contención y selecciona el número de ranuras de forma pseudo aleatoria a partir del intervalo $\left[0,2^{i} \mathrm{CW}\right.$ min $]$ donde ${ }^{i}$ especifica el número de retransmisiones en el caso que existan más colisiones en otras etapas de contención, si el paquete llega al máximo de retransmisiones este paquete se descarta.[15]

En la Fig. 1. se muestra un esquema para una transacción de un paquete con el IEEE802.11.

Fig. 1. TRANSACCIÓN DE UN PAQUETE DE DATOS CON EL ESTÁNDAR IEEE802.11

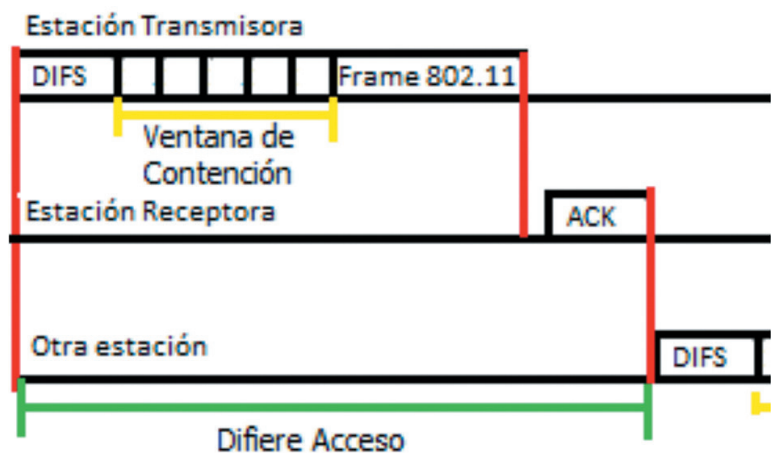

Fuente: Estándar IEEE 802.11 [15] 


\section{Desempeño de la capa física del IEEE802.11 sobre redes de largo alcance.}

El desempeño de la capa física sobre redes de largo alcance se explica en función de los límites que impone el nivel de sensitividad en la recepción de los radios WiFi comerciales y la máxima PIRE (Potencia Isotrópica Radiada Equivalente). Después se introduce la presencia de FER (Frame error rate) debida a la relación RSS (Receive signal Strength) y el nivel de ruido SNR (Signal to Noise ratio).

El límite que impone la capa física del IEEE802.11 para enlaces punto a punto de largo alcance está relacionado con la máxima PIRE la cual está regulada en cada país, el nivel de sensitividad que impone el radio para cada tipo de modulación y las pérdidas presentes por propagación.

En el caso de Colombia la regulación que existe en la implementación de radio enlaces punto a punto en la banda de $2.4 \mathrm{GHz}$ es una adaptación de la FCC, sección 15.247 (Regulations for Low Power, Non-Licensed Transmitters). La FCC impone una restricción de $30 \mathrm{dBm}$ de potencia transmitida con una antena de 6dBi PIRE; además por cada $3 \mathrm{dBi}$ adicionales de ganancia en la antena la potencia del transmisor se debe reducir en $1 \mathrm{dBm}$. [4][16]

Para predecir las pérdidas por propagación y determinar el nivel de señal recibida en el receptor se puede determinar a partir la ecuación de Friis considerado un margen de desvanecimiento. Este margen se origina en problemas de alineación, pérdidas en los conectores, cables, orografía del terreno, fenómenos meteorológicos como lluvia o nubosidad y la atenuación por árboles, un valor de desvanecimiento adecuado permite asegurar la estabilidad del radio enlace en el tiempo, aspecto fundamental para conectar emplazamientos distantes en varios kilometros. Otro modelo más apropiado para el cálculo de las pérdidas por propagación sobre este tipo de emplazamientos es el (ITM Irregular Terrain Model/ Longley Rice), el cual considera los fenómenos de reflexión y diffracción sobre la topografia del terreno. [17] [18][19]

En la Fig. 2 se presenta los límites de distancia en función de la ganancia de las antenas para un enlace punto a punto en la banda $2.4 \mathrm{GHz}$ según la FCC 15.247. Para el cálculo de las pérdidas por propagación se consideró el modelo de Friis con margen de desvanecimiento de $20 \mathrm{~dB}$ y el umbral de recepción se tomó de las especificaciones del radio XR2 (Se considera este radio por su bajo nivel de sensitividad) para cada velocidad de transmisión [6] [20] [21].

Fig. 2. LÍMITE DE DISTANCIA ESTÁNDAR IEEE802.11 CONSIDERNADO EL NIVEL DE SENSITIVIDAD DEL RADIO XR2

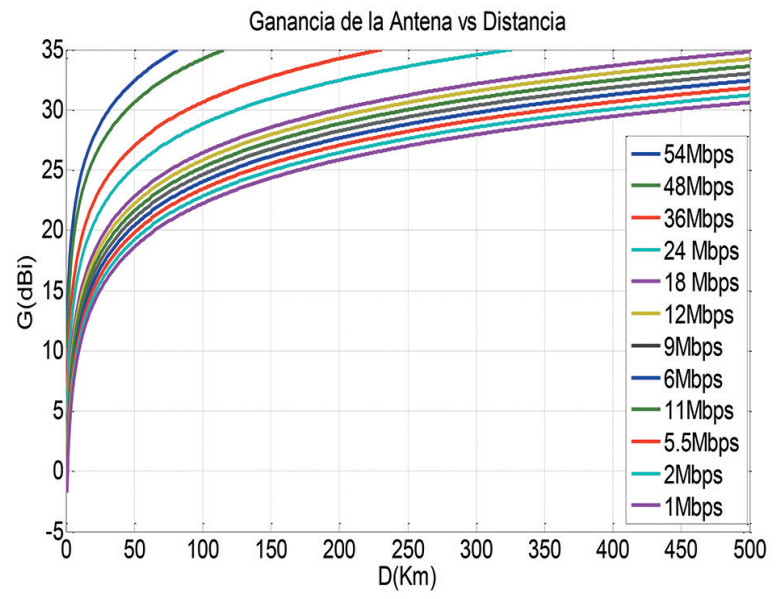

Fuente: Autor del proyecto

FER (FRAME ERROR RATE) en el IEEE802.11 para enlaces de largo alcance.

Las variaciones en las pérdidas de frames en enlaces de largo alcance se pueden clasificar en dos patrones o categorías de pérdidas. La primera de ellas es del tipo burst (generados principalmente por interferencias externas) y la segunda se atribuye a pérdidas residuales. [13]

Los enlaces IEEE802.11 en áreas rurales, por lo general, presentan bajo nivel de interferencias y, por lo tanto, las pérdidas tipo burst son despreciables en estos sitios. Además si la relación señal ruido se encuentra en el margen donde el $B E R<1 e-5$ se puede considerar que las pérdidas residuales son despreciables.

Características del FER vs. SNR en enlaces IEEE 802.11 de largo alcance.

A continuación se presentan las características del FER:

- La dependencia del FER (frame error rate) con respecto a la relación señal a ruido es muy cercana a su valor teórico para el IEEE802.11b/g. En el estándar IEEE 802.11b/g existe una pequeña ventana donde si el SNR se encuentra entre 4 a $6 \mathrm{~dB}$, el BER es aproximadamente 
del $100 \%$, y por encima de esta ventana la tasa de error es menor que el 1\%.[22][23]

- $\quad$ La FER no depende directamente de la distancia entre los nodos, solamente de la relación señal a ruido. [22][23][24]

- Existe una definitiva dependencia entre el FER y cada velocidad de transmisión.[22][23]

Considerando el modelo de la capa física del IEEE802.11b presentado en [25][26] y definiendo un nivel de ruido térmico de $-101.7 \mathrm{dBm}$ con un ancho de canal de $20 \mathrm{MHz}$, se calcula el número de paquetes recibidos en función del RSS (Received Signal Strength) para las diferentes velocidades de transmisión, mediante una MPDU (MAC protocol data unit) de 1094 bites, enviando 200 paquetes en broadcast, bajo un canal AWGN (Additive White Gaussian Noise). En la Fig. 3 se presenta el número de los paquetes recibidos comparado con el nivel de señal recibida para las velocidades de transmisión del estándar IEEE802.11b.

Fig. 3. PAQUETES RECIBIDOS VS. EL NIVEL DE SEÑAL RECIBIDA CONSIDERANDO UN NIVEL DE RUIDO DE -101.7DBM.

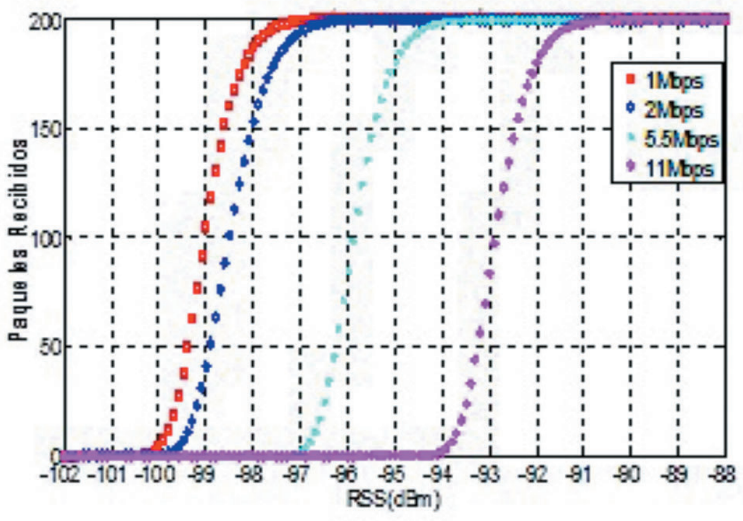

Fuente: Autor del proyecto.

Como se puede apreciar en la Fig. 3 la ventana de vulnerabilidad varía entre 4 y $5 \mathrm{~dB}$ para los cuales el FER puede variar de $1-100 \%$ como se expresó en las características de FER lo cual no depende de la longitud del enlace si no del nivel de señal recibida.

\section{Desempeño de la capa MAC del IEEE802.11 para enlaces de largo alcance.}

El desempeño de la capa MAC del IEEE802.11 sobre enlaces de largo alcance está expresado en función de algunos tiempos que definen el mecanismo de acceso al medio y que llevan implícitamente el tiempo.

La capa MAC no impone restricciones al límite de distancia existente entre los nodos de una manera explícita, pero si algunos de sus parámetros lo llevan de manera implícitamente como son el DIFS, Slottime, ACKtimeout, aunque en la versión más reciente del estándar se define que el máximo AirPropagationTime (dos veces el tiempo de propagación) es de, $1 \mu \mathrm{s}$ es decir. El estándar está diseñado para una red de área local, aunque en dicha versión del estándar se introduce el parámetro coverage class permite incrementar el valor de AirPropagationTime a $93 \mu$ s lo que permitiría concebirlo para una distancia de km.[15][21]

A continuación se presentan los parámetros más incidentes que expresan características del control de acceso al medio del estándar IEEE802.11 sobre redes de largo alcance.

ACKTimeout: Es el intervalo de tiempo que una estación transmisora debe esperar para recibir un ACK que confirma que la transmisión fue exitosa. Si no se recibe una confirmación dentro de ese intervalo de tiempo la estación transmisora considera que la transmisión fue fallida y vuelve a invocar el algoritmo backoff para realizar otra transmisión. Para enlaces de larga distancia si el valor del ACKTimeout es menor que dos veces el tiempo de propagación se generan retransmisiones innecesarias debido a que este expira, por lo tanto, el valor de ACKTimeout $>2 \delta$ para utilizar el canal de transmisión de una manera más conveniente de acuerdo a la distancia.[4][15][21]

DIFS: Es el tiempo durante el cual una estación debe sensar el canal libre antes de programar una nueva transmisión o reactivar la cuenta regresiva de la ventana de contención. Para un enlace de largo alcance la estación transmisora puede determinar que el canal está libre he inicializar el algoritmo de backoff sin estarlo debido a los tiempos de propagación, por lo tanto, este parámetro deberá ser incrementado por lo menos en un Round trip time; además se puede garantizar que las estaciones que comparten el medio no colisionen con los ACK en el caso de que existan más de dos estaciones. [4][15][21]

Slottime: Este parámetro incide directamente sobre la probabilidad de colisión entre las esta- 
ciones que comparten el medio. Este parámetro está definido dentro del estándar de tal forma que las estaciones que desean acceder al medio pueden colisionar si transmiten en un mismo slot, es decir, si una estación se encuentra en un slot previo puede determinar que la otra estación ha accedido al medio y evitar la colisión. Para considerar cómo el Slottime define la probabilidad de la colisión en función de la distancia deberemos considerar el intervalo de vulnerabilidad el cual es el periodo de tiempo durante el cual pueden ocurrir colisiones. Esto se debe a que la transmisión y recepción no son mecanismos instantáneos, es decir, éstos dependen del tiempo de propagación de la señal electromagnética entre las estaciones, el tiempo implementado en los mecanismos CS/ CCA y el tiempo en que la capa física cambia de modo recepción y comienza a transmitir el primer símbolo. El intervalo de vulnerabilidad se describe con el siguiente ejemplo; cuando una estación comienza a transmitir datos, éstos no podrán ser detectados por las otras estaciones de manera instantánea por lo tanto pueden considerar que el canal está libre y comenzar a transmitir y/o generar colisiones. Las estaciones solamente podrán determinar que el canal está ocupado después de un determinado tiempo el cual debe ser, por lo menos, el periodo de vulnerabilidad.[4][15][21] [27]

Periodo de vulnerabilidad es igual a la suma de:

- El tiempo que le toma a la estación transmisora evaluar el canal y de notificar ese estado a la capa MAC.

- El tiempo que tarda una estación destino cambiar de estado recepción al de transmisión.

- El tiempo de propagación.

Cuando se utiliza el Slottime definido en el estándar 20 y $9 \mu \mathrm{s}$ para IEEE802.11b/g respectivamente, al incrementar la distancia el intervalo de vulnerabilidad aumenta debido número de slots que encajan dentro del tiempo de propagación, es decir, la probabilidad de colisión entre las estaciones aumenta. [4][15][21]

Si se considera el valor del Slottime de tal forma que sea igual al intervalo de vulnerabilidad (slottime $\approx 2 \delta$ ) las estaciones colisionarían solamente si transmiten en un mismo slot como se puede apreciar en la Fig. 4, por lo tanto, los retardos y paquetes perdidos disminuyen.
Fig. 4. EL VALOR DEL SLOTTIME ES MAYOR O IGUAL A DOS VECES EL TIEMPO DE PROPAGACIÓN

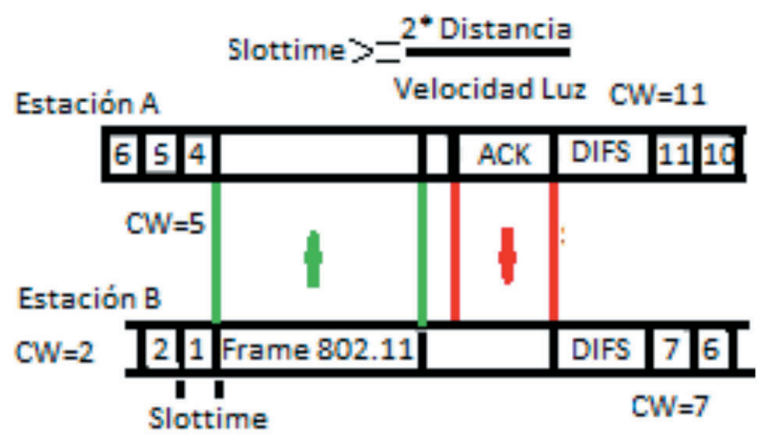

Fuente: Adaptado por los autores de [6] [21] [28]

Si el valor del Slottime es menor pero comparable con el intervalo de vulnerabilidad (slottime $\approx \delta$ ) las estaciones podrán colisionar si transmiten en slots contiguos, el enlace pierde la simetría como se puede ver en la Fig. 5, el throughput se maximiza pero aumentan los retardos y los paquetes perdidos. Para un valor de slottime $<\delta$ se incrementa el intervalo de vulnerabilidad el número de colisiones aumenta, los retardos se incrementan y el throughput disminuye.

Fig. 5. EL VALOR DEL SLOTTIME ES CERCANO AL TIEMPO DE PROPAGACIÓN

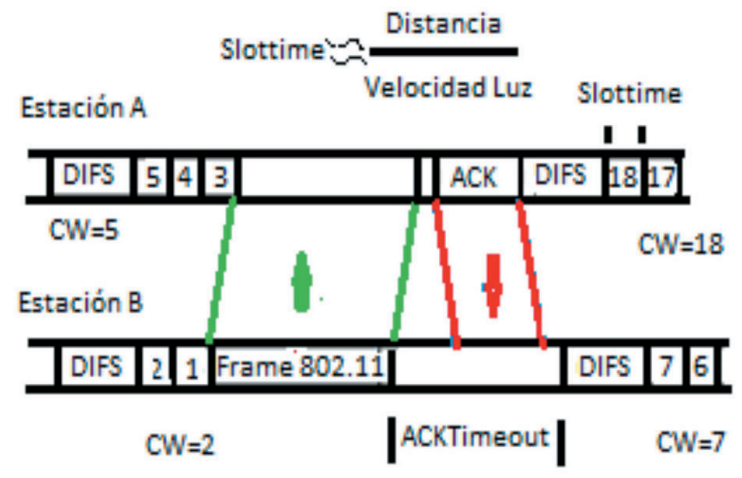

Fuente: Adaptado por los autores de [6] [21] [28]

\section{E. Modelos para calcular el throughput}

Para calcular el throughput UDP unidireccional sobre el IEEE802.11 en función de la distancia se basan en modelos propuestos para redes de área local [29][30]. Para calcular el máximo throughput UDP unidireccional ( $\left.T R_{U D P U}\right)$ se utiliza la siguiente expresión:

$$
T P_{U D P U}=\frac{L_{\text {Paquete } \_ \text {UDP }}}{T_{\text {Trans } \_ \text {UDP }}}
$$


Donde $L_{\text {Paquete_UDP }}$ se refiere al tamaño del paquete UDP.

La $T_{\text {Trans UDP }}$ se considera como el tiempo que transcurre una transacción UDP sobre el estándar IEEE802.11, el envío de un paquete UDP se describe en la Fig. 6.

Fig. 6. ESQUEMA SIMPLIFICADO DE UNA TRANSACCIÓN UDP SOBRE IEEE802.11

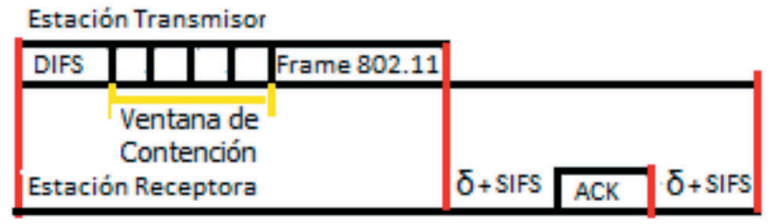

Fuente: Autor del proyecto

Primero la estación transmisora verifica que el canal se encuentre libre durante un $T_{D I F S}$ para inicializar la ventana de contención, la cual se disminuye hasta que la ventana llega a cero la estación. Se procede luego a enviar el paquete UDP y cuando la estación llamada receptora lo recibe espera un tiempo $T_{S I F S}$ para confirmar el arribo del paquete con un ACK_MAC. [103]

El tiempo para transmitir un segmento UDP sobre el estándar IEEE802.11b/g considerados los tiempos de propagación está dado por la siguiente expresión:

$$
\begin{aligned}
T_{\text {Trans_UDP }}= & T_{\text {DIFS }}+T_{w_{\text {_contención }}}+T_{\text {Datos UDP }} \\
& +T_{\text {SIFS }}+T_{802.11 \_A C K}+2 * \delta(2)
\end{aligned}
$$

Cada uno de los tiempos que hacen parte de la ecuación (2) se describirán a continuación.

El tiempo $T_{D I F S}$ espacio intertrama de DCF se presenta a continuación la siguiente expresión.

$$
T_{\text {DIFS }}=2 * T_{\text {Slottime }}+T_{\text {SIFS }}
$$

Para calcular el tiempo que transcurre en la etapa contención $T_{w_{\text {_contención }}}$ se considera que el canal de comunicación se encuentra libre de interferencias y el nivel de señal recibida se encuentra por encima del nivel de sensibilidad en el receptor que se define para cada velocidad de transmisión, es decir, el $\mathrm{BER}=0$ (Bit error rate), por lo tanto, se considera un canal de comunicación ideal, el $\mathrm{FER}=0$, es decir, las transmisiones son exitosas, por lo tanto la variable pseudo aleatoria con distribución uniforme de la ventana de contención es seleccionada del intervalo de $\left(0, C W_{\text {min }}\right)$ donde el tiempo promedio de la ventana de contención está dado por la siguiente expresión.

$$
T_{W_{\text {_contención }}}=\frac{C W_{\text {min }}}{2} * T_{\text {Slottime }}
$$

Para el cálculo del tiempo que transcurre al enviar un paquete de datos $T_{\text {Datos_UDP }}$ utilizamos la siguiente expresión.

$$
T_{\text {Datos_UDP }}=T_{\text {Preambulo }}+\frac{L_{M P D U_{-} \text {frame }}}{R_{\text {Tasa }}} * 8
$$

Para el IEEE802.11b.

El $T_{802.11 \_A C K}$ es el tiempo que transcurre para que la estación receptora envíe un ACK.

$$
T_{802.11 \_A C K}=T_{\text {Preambulo }}+\frac{L_{802.11 \_A C K}}{R_{\text {Basic }}} * 8
$$

$\delta$ Se refiere al tiempo que tarda en viajar la señal electromagnética entre las dos estaciones.

$$
\delta=\frac{d}{v}=\frac{d(\mathrm{~km})}{3 e 5(\mathrm{~km} / \mathrm{s})}
$$

Al utilizar el estándar IEEE802.11g $T_{\text {Datos_UDP }} \mathrm{y}$ $T_{\text {802.11_ACK }}$ se convierte en la ecuación 7 y 8 .

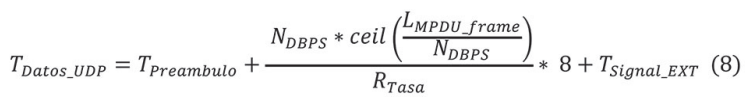

$T_{802.11 \text { _ACK }}=T_{\text {Preambulo }}+\frac{N_{\text {DBPS }} * \operatorname{ceill}\left(\frac{L_{802.11 \text { ACCK }}}{N_{\text {DBPS }}}\right)}{R_{\text {Basic }}} * 8+T_{\text {Signal_EXT }}(9)$

Con la ecuación (1), considerado un paquete UDP de 1440bites y utilizado el estándar IEEE802.11b a 11Mbps, un canal de comunicación ideal, es decir, el BER $=0$ y que los temporizadores del ACK no expiran y los demás parámetros tomados de la Tabla II, en la Fig. 6 se presenta el throughput UDP unidireccional en un enlace punto a punto, estos resultados se pueden comparar con las medidas obtenidas con el emulador de canal Spirent 5500 [12] [31]. 
TABLA II

Valores para los parámetros en el modelo UDP Unidireccional

\begin{tabular}{|c|c|c|c|c|}
\hline Parámetro & Definición & $\begin{array}{l}\text { Valor } \\
\mathbf{8 0 2 . 1 1 b}\end{array}$ & $\begin{array}{l}\text { Valor } \\
\mathbf{8 0 2 . 1 1 g}\end{array}$ & Unidad \\
\hline$C W_{- \text {min }}$ & $\begin{array}{c}\text { Tamaño de la } \\
\text { ventana contienda } \\
\text { mínima }\end{array}$ & 31 & 15 & Slots \\
\hline$T_{\text {Slottime }}$ & $\begin{array}{l}\text { Tiempo asignado } \\
\text { al slot. }\end{array}$ & 20 & 9 & $\mu s$ \\
\hline$T_{w_{-} \text {contención }}$ & $\begin{array}{l}\text { Tiempo promedio } \\
\text { de la ventana } \\
\text { contienda. }\end{array}$ & 310 & 67 & $\mu s$ \\
\hline$T_{D I F S}$ & Tiempo DIFS & 50 & 28 & $\mu s$ \\
\hline$T_{S I F S}$ & Tiempo SIFS & 10 & 10 & $\mu s$ \\
\hline$T_{\text {Preambulo }}$ & $\begin{array}{l}\text { Duración PLCP } \\
\text { Largo/Corto }\end{array}$ & $192 / 96$ & 20 & $\mu s$ \\
\hline$L_{\text {MPDU_frame }}$ & $\begin{array}{l}\text { SERVICE+ MAC+ } \\
\text { SNAP/LLC+ IP+ } \\
\text { UDP+ DATOS+ FCS }\end{array}$ & $\begin{array}{c}28+8+ \\
20+8+ \\
1440+0 \\
=1504\end{array}$ & $\begin{array}{c}28+8+ \\
20+8+ \\
1440+4 \\
=1508\end{array}$ & Bites \\
\hline$R_{\text {Tasa }}$ & $\begin{array}{c}\text { Tasa de Transmisión } \\
\text { Capa física }\end{array}$ & $\begin{array}{c}1,2 \\
5.5,11 .\end{array}$ & $\begin{array}{c}6,9 \\
12,18, \\
24,36, \\
48,54\end{array}$ & Mbps \\
\hline$R_{\text {Basic }}$ & $\begin{array}{c}\text { Tasa de transmisión } \\
\text { capa física ACK. }\end{array}$ & 1,2 & $\begin{array}{c}6,9,12 \\
18,24\end{array}$ & Mbps \\
\hline$T_{\text {Signal_EXT }}$ & Extensión de Señal & - & 6 & $\mu s$ \\
\hline$L_{802.11 \_A C K}$ & $\begin{array}{l}\text { Longitud del frame } \\
802.11 \text { Ack }\end{array}$ & 14 & 14 & Bites \\
\hline$N_{D B P S}$ & $\begin{array}{c}\text { Número de data bits } \\
\text { por símbolo para } \\
\text { OFDM. }\end{array}$ & - & $\begin{array}{c}216,192 \\
144,96 \\
72,48 \\
36,24 .\end{array}$ & Bits \\
\hline
\end{tabular}

Fuente: Autor del proyecto.

Fig. 7. THROUGHPUT UDP UNIDIRECCIONAL ESTÁNDAR IEEE802.11B A 11MBPS

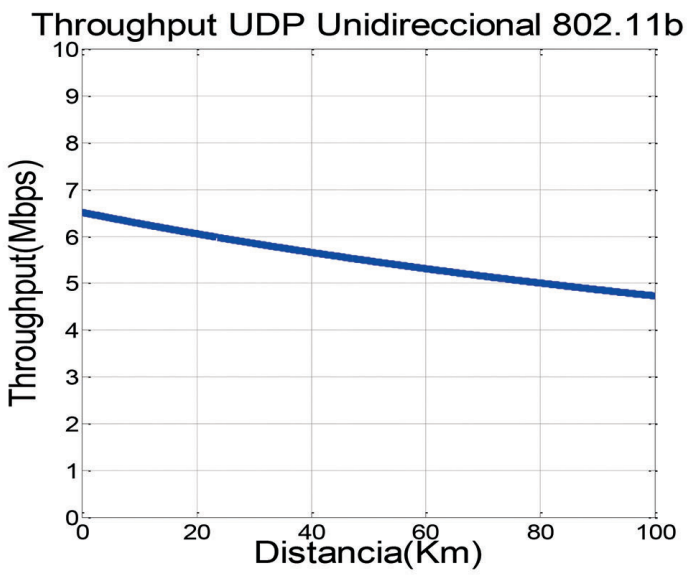

Fuente: Autor del proyecto

Este mismo análisis se puede utilizar para calcular el throughput UDP unidireccional con los protocolos que realizan una mejor utilización del canal de comunicación, como son el protocolo bursting donde elimina el llamado consecutivo al algoritmo backoff y fastframing el cual concatena tramas para aumentar el tamaño de la MPDU.
Para el cálculo del throughput UDP saturado Bidireccional se puede utilizar el modelo de Bianchi (El cual se basa en la cadena bidimensional de Markov) siempre que se considere que el slottime $\geq 2 \delta$ para garantizar que las estaciones puedan colisionar solamente si transmiten en un mismo slot.

A continuación en la Fig. 8 se presenta el throughput UDP Bidireccional para un el enlace punto a punto $\mathrm{N}=2$ con el modelo de Bianchi [32] considerado slottime $=2 \delta$, no se desprecia que la colisión entre dos estaciones genera que la estación tenga que esperar $T_{\text {SIFS }}$ más el $T_{\text {ACKTimeout }}=2 \delta$ para evitar que el temporizador de ACK expire, este modelo define un $B E R=0$ (esto significa que el nivel de recepción siempre se encuentra mayor que el nivel de sensitividad del receptor para la respectiva velocidad de transmisión), una MPDU de 1500bites. El resultado de throughput Bidireccional en un enlace punto a punto se presenta en la Fig. 8. [32][33]

Fig. 8. THROUGHPUT UDP BIDIRECCIONAL ESTÁNDAR IEEE802.11B A 1, 2, 5.5 Y 11MBPS.

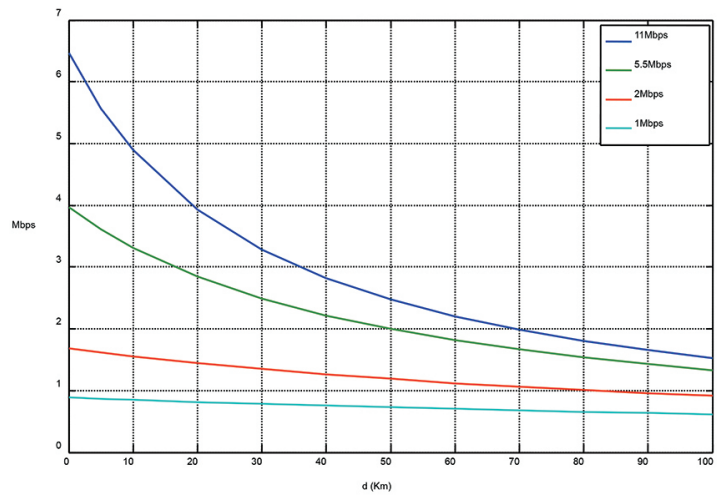

Fuente: Autor del proyecto.

Cuando el valor de slottime $<2 \delta$ el modelo de Bianchi [32] y Tinnirello [33] se invalida y debe considerar el modelo propuesto por J. Simo [21] que permite predecir el throughput UDP en redes de largo alcance su modelo identifica la ocurrencia de las colisiones a partir del intervalo de vulnerabilidad donde una estación puede colisionar con la transmisión de otra estación debido a que ésta no puede escuchar el arribo del paquete proveniente de la otra estación. Para calcular la probabilidad que al menos una estación transmita en un slot se toma parte del modelo de Bianchi mientras que 
para determinar la probabilidad de que ocurra una colisión en otro slot se toma el modelo J. Simo [21] el cual lo calcula a partir del intervalo de vulnerabilidad que se genera por el número de slottime(s) que transcurren sin percibir la transmisión de otra estación.

Analizadas las características que presenta el modelo J. Simo [21] en cuanto al $B E R=0$ se construyó el modelo "CPS" como aproximación para el cálculo del throughput UDP saturado Bidimensional en enlace punto a punto IEEE802.11 este modelo se obtuvo a partir de la máquina de estados que se presenta en la Fig. 9 en la cual se define el tiempo de colisión, de una transmisión exitosa y la ventana donde ocurre una colisión.

A partir de esta máquina de estados se determina el tiempo de transmisión exitosa y el tiempo de colisión considerado el algoritmo backoff el cual genera un retardo que depende del estado del canal ya sea que se encuentre libre u ocupado y el número de retransmisiones generadas por las colisiones, los anteriores tiempos se utilizan en la evaluación del throughput en el enlace de comunicación.

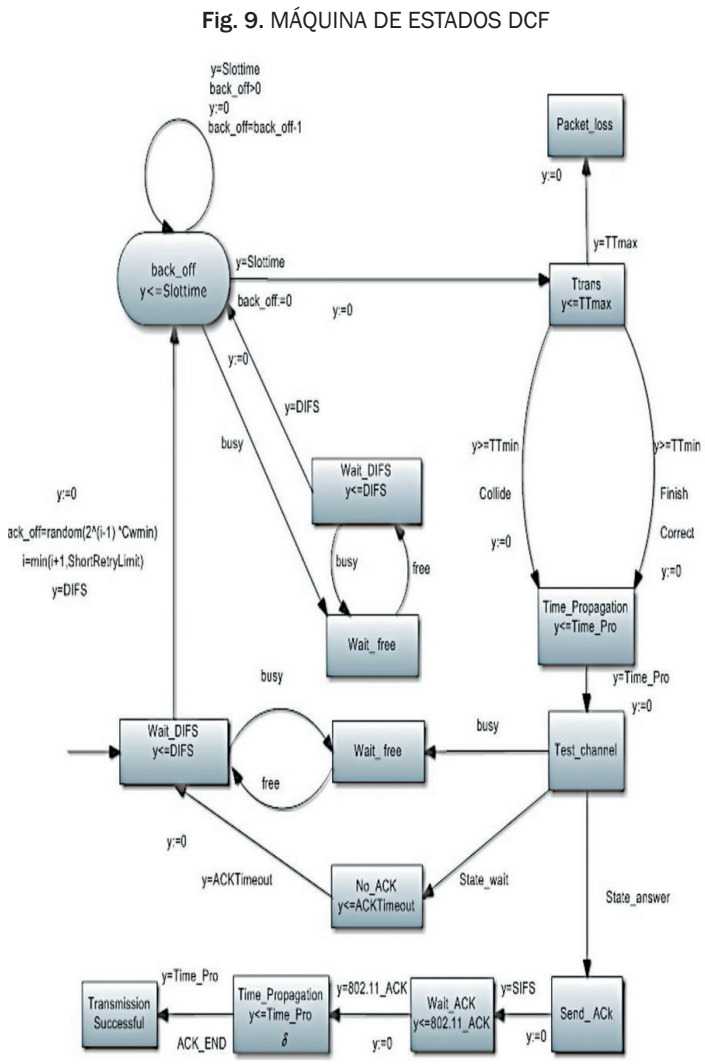

Fuente: Adaptado de Probabilistic Model Checking of the IEEE 802.11 Wireless Local Area Network Protocol [34] y A Finite State Model for IEEE 802.11 Wireless LAN MAC DCF [35]
Para diferenciar en cuáles casos se llega al estado de transmisión exitosa y en cuáles ocurre una colisión se toman consideraciones presentadas en el modelo J. Simo en la definición intervalo del IV (intervalo de vulnerabilidad), para definir el intervalo de colisión (IC), definido como el intervalo de tiempo durante el cual la estaciones STA y STB, que hacen parte del enlace punto a punto, pueden colisionar cuando transmitan asumiendo que STA tiene una ventana $C W_{a}$ y $\mathrm{CW}_{\mathrm{b}}$ STB tiene una ventana, con un valor de Slottime fijo. Para una mejor comprensión, considérese el siguiente ejemplo. Dos estaciones STA y STB intentan transmitir de manera simultánea. La ventana seleccionada de forma pseudo aleatoria para STA es $C W_{a}$ y para STB es $\mathrm{CW}_{\mathrm{b}}$. La estación que tenga la menor ventana de contención transmite, pero debido a la distancia entre ellas, la otra estación no detecta con su mecanismo CC/CCA que el canal se encuentra ocupado. Si se supone que STA tiene la menor ventana, sólo podrá colisionar con STB cuando la ventana de contención llegue a cero debido a que no percibe la transmisión de STA, lo cual se puede calcular a partir del número de Slottime(s) que transcurren en el tiempo de propagación y el tiempo que tarda el mecanismo CCA en determinar que el canal se encuentra ocupado, $I C=\delta+C C A T i m e$. También se puede calcular el número de slots dentro de los cuales puede ocurrir una colisión el cual se nombra como ICN (Intervalo de colisión normalizado), este se encuentra expresado en la siguiente ecuación:

$$
I C N=\left(\frac{\delta+\text { CCATime }}{T_{\text {Slottime }}}\right)
$$

Considerado que la ventana de contención se encuentra ranurada el intervalo de colisión se aproxima a un valor entero, el intervalo de colisión es la mitad del intervalo de vulnerabilidad definido por J. Simo [21].

Conocido el número de slots que puede transcurrir antes que alguna estación determine que el canal se encuentra ocupado debido a la distancia presente entre estas dos estaciones y con el valor de las ventanas de contención $C W_{a}, C W_{b}$ se determina la ocurrencia de colisión. De acuerdo a lo anterior se considera que una colisión existe si:

$$
\operatorname{abs}\left(\mathrm{CW}_{\mathrm{a}}-\mathrm{CW}_{\mathrm{b}}\right) \leq \mathrm{ICN}
$$


Para calcular el throughput en un enlace punto a punto a partir de una secuencia de estados que describa el envío de cada paquete se debe determinar a partir del cociente entre la cantidad de paquetes enviados por las dos estaciones multiplicado por el tamaño promedio de los paquetes entre el tiempo que transcurre para realizar el envío de estos.

El throughput UDP Bidireccional saturado () lo podemos calcular a partir del cociente entre el número de los paquetes enviados por las dos estaciones y el tiempo promedio que transcurre en enviarlos considerando el número de intentos $\mathrm{NI}$ el cual depende del número de colisiones, en la siguiente ecuación se presenta una expresión para el cálculo de esta métrica de red.

Ecuación (12):

$$
T R_{U D P B}=\frac{\sum_{i=0}^{N E} L_{P a q u e t e \_U D P}}{\sum_{i=0}^{N E} T_{\text {exitosa }}+\sum_{i=0}^{N I-N E} T_{\text {colision }}+\sum_{i=0}^{N I} T w(i)}
$$

El promedio para el tiempo una transmisión exitosa y la de una colisión considerado que la estación transmite se expresa en la ecuación 13 y 14 respectivamente. [6][21][32][33]

$$
\begin{aligned}
& T_{\text {exitosa }}=T_{\text {DIFS }}+T_{\text {Datos }} \text { UDP } \\
& T_{\text {colision }}=T_{\text {SIFS }}+T_{802.11_{\text {IIAS }}}+T_{\text {Datos }_{\text {UDP }}}+T_{\text {ACKTimeout }}+T_{\text {Slottime }}
\end{aligned}
$$

Donde:

$$
T_{\text {ACKTIMEOUT }}=T_{S I F S}+T_{802.11 \_A C K}+2 * \delta \text { (15) }
$$

Como las expresiones (13) y (14) no dependen de $i$ y se considera que el tamaño del paquete UDP es el mismo para cada transmisión la ecuación (12) se reescribe de la siguiente forma:

Ecuación (16):

$$
T R_{U D P B}=\frac{L_{\text {Paquete }_{U D P}}}{T_{\text {exitosa }}+\left(\frac{N I}{N E}-1\right) T_{\text {colision }}+\frac{1}{N E} \sum_{i=0}^{N I} T w(i)}
$$

Donde:

$$
T w(i)=\min \left(T_{w_{R(i), 1}} T_{w_{K(i), 2}}\right)
$$

Define el tiempo promedio de la ventana de contención para los paquetes enviados por las dos estaciones $T_{W R(i), 1}$ para ST1 (Estación 1) y $T_{W K(i), 2}$ para ST2 (Estación 2) la cual depende de la ocurrencia de colisión según (11) y el número de retransmisiones de ST1 y ST2. La función min permite decir cuál de las dos estaciones genera el evento de una transmisión exitosa o la ocurrencia de una colisión entre las dos estaciones.

$$
\begin{aligned}
T_{w_{R(i), 1}} & =\left(C W_{R(i), 1} * T_{\text {Slottime }}\right)(18) \\
T_{w_{K(i), 2}} & =\left(C W_{k(i), 1} * T_{\text {slottime }}\right)(19)
\end{aligned}
$$

La ventana de contención ranurada es entera y se selecciona de forma pseudo aleatoria a partir de una distribución uniforme como se presente en las siguientes expresiones.

$$
\begin{aligned}
& C W_{R(i), 1}=\operatorname{rand}\left(0,2^{R(i)} * C W_{\text {min }}\right) \\
& C W_{K(i), 2}=\operatorname{rand}\left(0,2^{k(i)} * C W_{\text {min }}\right)
\end{aligned}
$$

Los estados para la estación 1 y 2 se definen a partir de las variables aleatorias $R(i)$ y $K(i)$ respectivamente, estas dependen del estado transmisión, es decir, backoff=0, el canal ocupado o bussy donde congela el contador de backoff por sensar que el canal se encuentra ocupado, el estado de colisión dobla la ventana de contención debido, el estado de reinicio de la ventana de contención por transmisión exitosa o pérdida de paquete se logra cuando llega al número máximo de retransmisiones, los valores que pueden tomar las variables aleatorias se expresan a continuación.

$$
R(i), K(i)=\{0,1,2 \ldots ., N R\}(22)
$$

Donde $N R$ es el número máximo de retransmisiones en el estándar IEEE802.11b/g es 7 y 4 cuando se utiliza el servicio Request to send RTS / Clear to send CTS.

A continuación en la Fig. 10, se presenta una comparación de la predicción del throughput UDP saturado bidireccional que presenta el modelo de J. Simo y el modelo CPS para un enlace punto a punto considerando los valores definidos en la Tabla II, un tamaño de paquete UDP de 1000bites y para distancias entre 0 a $100 \mathrm{Km}$.

Fig. 10. COMPARACIÓN DEL MODELO DE J. SIMO CON EL MODELO CPS

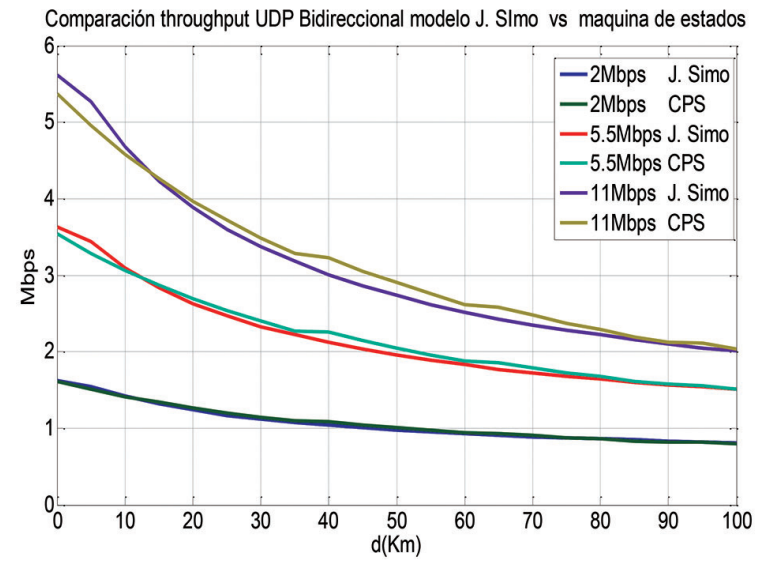

Fuente: Autor del proyecto. 


\section{MEDICIONES DE THROUGHPUT}

Para las mediciones experimentales de throughput se diseñó un grupo de prototipos Wi-Fi autónomos alimentados con energía fotovoltaica, los cuales se presentan en la Fig. 11, los cuales se utilizaron para las pruebas de laboratorio y en campo abierto.

Fig. 11. PROTOTIPOS DE NODO WI-FI AUTÓNOMO ALIMENTADO CON PANELES FOTOVOLTAICOS
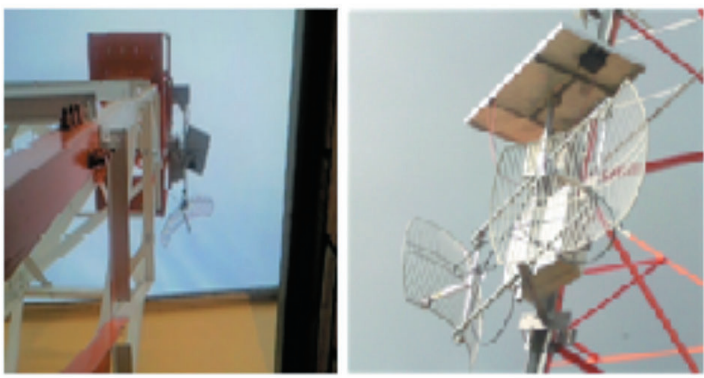

Fuente: Autor

Cada enlace punto a punto se realizó con las motherboards Soekris NET4801-48 y ALIX2D2 en las cuales se le instaló el sistema operativo Linux Voyage y el driver MADWIFI. Cada uno de los nodos utiliza el radio EXTREMErange2 (chipset atheros AR5414). En la Tabla III se dan las especificaciones de las características técnicas del hardware utilizado para la construcción de cada uno de los nodos que hacen parte del enlace de comunicación.

TABLA III

Hardware utilizado para las pruebas

\begin{tabular}{|c|c|c|}
\hline Dispositivo & Referencia & Características \\
\hline Motherboard 1 & ALIX2D2 & $\begin{array}{l}\text { CPU: } 500 \text { MHz, AMD Geode } \\
\text { LX800 } \\
\text { DRAM: } 256 \text { MB DDR (on } \\
\text { board ) } \\
\text { Storage: CompactFlash socket } \\
2 \text { miniPCI } \\
\text { Firmware: Award tinyBIOS }\end{array}$ \\
\hline Motherboard 2 & $\begin{array}{l}\text { Soekris } \\
\text { net4801-48 }\end{array}$ & $\begin{array}{l}\text { CPU: } 233 \mathrm{MHz}, \text { AMD Geode } \\
\text { SC1100 } \\
\text { SDRAM: } 128 \text { Mbyte (on board) } \\
\text { Storage: CompactFlash } \\
1 \text { Mini-PCl socket }\end{array}$ \\
\hline Radios & Ubiquiti (XR2) & $\begin{array}{l}\text { Chipset atheros AR5414 32-bit } \\
\text { mini-PCI Type IIIA }\end{array}$ \\
\hline Sistema Operativo & Linux Voyage & Version 0.6.2 \\
\hline Driver & MADWIFI & $\begin{array}{l}\text { madwifi-modules-2.6.30- } \\
\text { voyage_0.9.4 rc2-1+ } \\
\text { 7.0-1_i386.deb }\end{array}$ \\
\hline Antenas & HG2424 & $24 \mathrm{dBi}$ \\
\hline Cable & LMR400 & 2 metros \\
\hline
\end{tabular}

Fuente: Autor del proyecto.
Para las pruebas de laboratorio se uso un cable coaxial para emular las pérdidas por propagación en el enlace punto a punto considerando las distancias $0,5,10 \mathrm{Km}$.

Para cada nodo de comunicación se le ajustaron los tiempos de la capa MAC para mejorar el desempeño sobre redes de largo alcance, los parámetros modificados son el tiempo ACKtimeout el cual se incremento $2 \delta$ como se aprecia en la ecuación (23) y el slottime se aumentó en $\delta$ ver ecuación (24) de tal forma que el throughput bidireccional sea cercano al máximo, todo estos parámetros se ajustaron con el driver de MADWIFI el cual sirve como controlador sobre el chip Atheros AR5414 que hace parte del radio XR2.

$$
\begin{aligned}
& \text { ACKTimeout }=48(\mu \mathrm{s})+\frac{2 * d(\mathrm{~m})}{300(\mathrm{~km} / \mathrm{us})} \\
& \text { Slottime }=\text { Slottime_std }(\mu \mathrm{s})+\frac{d(\mathrm{~km})}{300(\mathrm{~km} / \mathrm{us})}
\end{aligned}
$$

Donde Slottime ${ }_{\text {std }}$ es el tiempo que se define en el estándar IEEE802.11b/g para un slot.

Las mediciones del throughput UDP en cada uno de los enlaces punto a punto IEEE802.11b/g realizados en laboratorio y en campo abierto se utilizó el generador de tráfico IPERF [36], cada prueba tuvo una duración de 180 segundos. Para los resultados de las pruebas realizadas en laboratorio se le introdujeron por software los retardos por propagación.

Para las pruebas de campo abierto se seleccionaron diferentes puntos en Bucaramanga y zonas circundantes al área metropolitana (zonas rurales) en los que se disponía de seguridad para los equipos de medición y los nodos Wi-Fi autónomos durante la permanencia de las pruebas.

TABLA IV

\begin{tabular}{|c|c|c|c|}
\hline Nodo & Latitud & Longitud & Altura (m) \\
\hline A & $7^{\circ} 8^{\prime} 26.09 " \mathrm{~N}$ & $73^{\circ} 7^{\prime} 17.87^{\prime \prime} 0$ & 991 \\
\hline B & $7^{\circ} 8^{\prime} 21.0^{\prime \prime} \mathrm{N}$ & $73^{\circ} 7 ' 16.1^{\prime \prime} 0$ & 990 \\
\hline C & $7^{\circ} 7{ }^{\prime} 59.81 " \mathrm{~N}$ & $73^{\circ} 7{ }^{\prime} 17.08^{\prime \prime} 0$ & 1000 \\
\hline D & $7^{\circ} 7{ }^{\prime} 39.90 " \mathrm{~N}$ & $73^{\circ} 7 ’ 15.80^{\prime \prime} 0$ & 998 \\
\hline E & $7^{\circ} 6$ '14.4”N & $73^{\circ} 5^{\prime} 10.5 " 0$ & 1293 \\
\hline $\mathrm{F}$ & $7^{\circ} 9^{\prime} 24.50 " \mathrm{~N}$ & $73^{\circ} 9^{\prime} 43.40^{\prime \prime} 0$ & 1060 \\
\hline G & $7^{\circ} 4^{\prime} 55.5^{\prime \prime} \mathrm{N}$ & $73^{\circ} 11^{\prime} 48.7^{\prime \prime} 0$ & 1358 \\
\hline $\mathrm{H}$ & $7^{\circ} 8^{\prime} 28.4^{\prime \prime} \mathrm{N}$ & $73^{\circ} 7 ' 23.1^{\prime \prime} 0$ & 975 \\
\hline I & $07^{\circ} 07^{\prime} 14.4 " \mathrm{~N}$ & $73^{\circ} 04^{\prime} 37.4^{\prime \prime} 0$ & 1565 \\
\hline
\end{tabular}

Sitios seleccionados para las pruebas de campo

Fuente: Autor del proyecto. 
En la figura 12 se presenta la ubicación de cada uno de los emplazamientos seleccionados.

Fig. 12. SITIOS SELECCIONADOS PARA LOS ENLACES DE COMUNICACIÓN

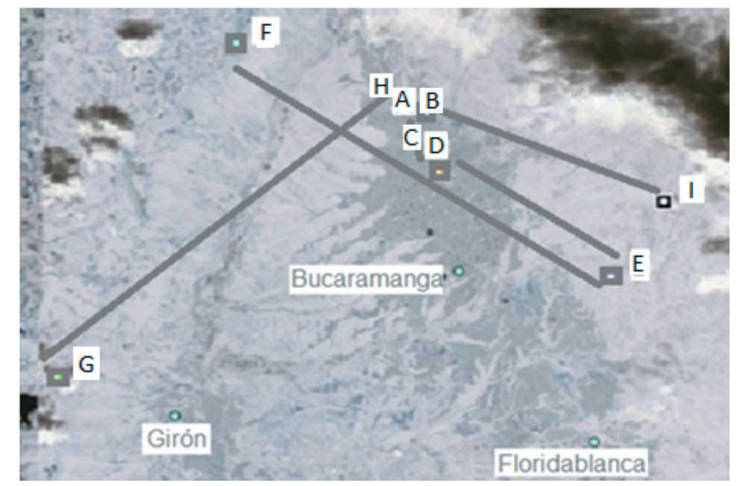

Fuente: Autor del proyecto

A continuación en la Tabla $V$ se describe los diferentes enlaces punto a punto construido en campo abierto y su respectiva configuración del ACKTimeout y el slottime.

TABLA V

Enlaces de comunicación establecidos en campo

\begin{tabular}{|l|c|c|c|c|}
\hline \multirow{2}{*}{ Enlaces } & \multirow{2}{*}{$\begin{array}{c}\text { Distancia } \\
(\mathrm{km})\end{array}$} & \multicolumn{2}{c}{ Slottime $(\mathrm{ms})$} & \multirow{2}{*}{$\begin{array}{c}\text { Acktimeout } \\
(\mathrm{ms})\end{array}$} \\
\cline { 3 - 4 } & & $11 \mathrm{~g}$ & $11 \mathrm{~b}$ & 48 \\
\hline A-B & 0.16 & 9 & 20 & 48 \\
\hline B-C & 0.65 & 9 & 20 & 75 \\
\hline D-E & 4.674 & 23 & 34 & 116 \\
\hline E-F & 10.212 & 43 & 54 & 79 \\
\hline B-I & 5.01 & 25 & 36 & 116 \\
\hline G-H & 10.46 & 43 & 54 & \\
\hline
\end{tabular}

Fuente: Autor del proyecto

En la Fig. 13 se presentan los resultados de las pruebas de throughput realizadas en campo abierto comparado con los valores obtenidos con el modelo teórico y las pruebas realizadas en laboratorio para cada una de las diferentes distancias.

En la Fig. 13 se puede apreciar que las pruebas de throughput UDP unidireccional en campo abierto se aproximan al modelo teórico y al valor obtenido en las pruebas de laboratorio, aunque se puede apreciar un discrepancia en las medidas obtenidas para la distancia de $5.01 \mathrm{Km}$ esto se debe a que en este lugar funcionan unos sistemas de comunicación que operan en la banda de 2.4GHz el cual se encuentra en el rango de frecuencias donde opera nuestro sistema de comunicación. En la Fig. 14 se presenta las mediciones del espectro en el rango de frecuencias 2412 a $2484 \mathrm{MHz}$, al analizar el espectro se puede apre- ciar la existencia de interferencia en el canal de comunicación, lo cual aumentó el número de paquetes medidos, esta medición se realizó sobre en el punto I de la Fig. 12.

Fig. 13. PRUEBAS DE CAMPO UDP UNIDIRECCIONAL IEEE802.11B

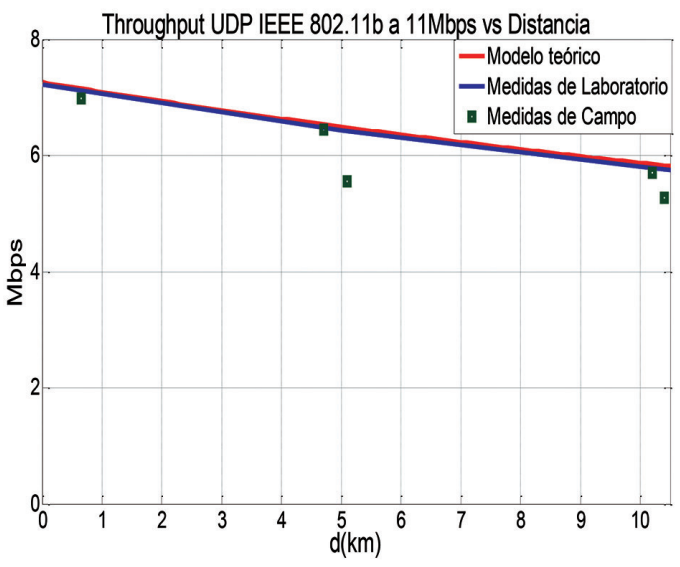

Fuente: Autor del proyecto.

Fig. 14. MEDICIÓN DEL ESPECTRO EN LA BANDA DE 2.4GHZ

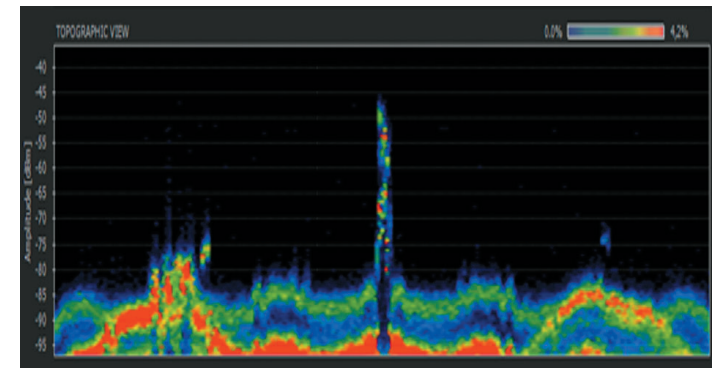

Fuente: Autor del proyecto

A continuación en la Fig. 15 se presenta el throughput UDP con la técnica bursting que se puede utilizar sobre el radio XR2.

Fig. 15. PRUEBAS DE CAMPO UDP UNIDIRECCIONAL BURSTING IEEE802.11B

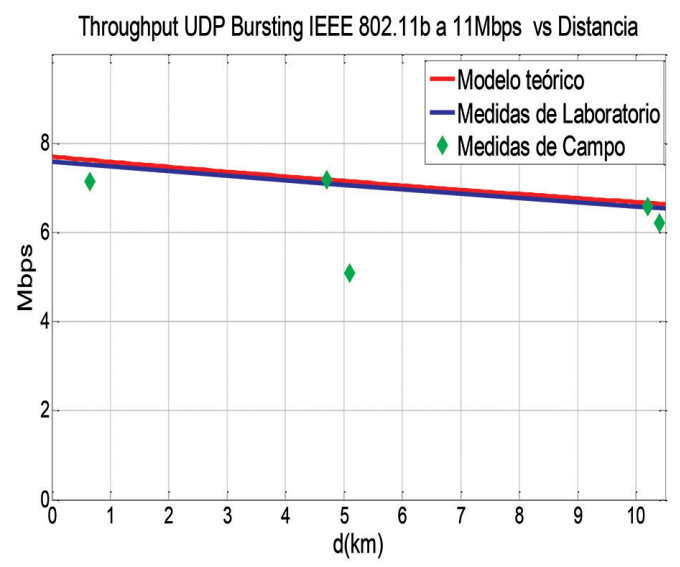

Fuente: Autor del proyecto 
En la Fig. 16 se presenta las mediciones de throughput UDP Bidireccional y el valor obtenido con el modelo teórico "CPS" propuesto. En la Fig. 16 se puede apreciar que el modelo permite predecir el throughput UDP que se obtuvo en el campo abierto.

Fig. 16. PRUEBAS DE CAMPO UDP BIDIRECCIONAL IEEE802.11B

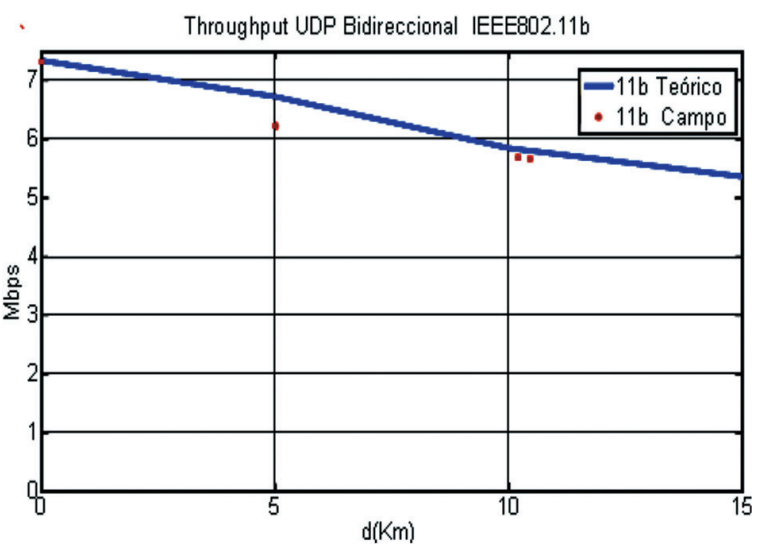

Fuente: Autor del proyecto

\section{CONCLUSIONES}

Se realizó e implementó una metodología para caracterizar el desempeño del estándar IEEE802.11 en un radio enlace de largo alcance, Donde se logró determinar el throughput UDP máximo que se puede obtener de acuerdo a la distancia presente entre las estaciones.

Los radios Wi-Fi modificados los parámetros de su control de acceso al medio se presentan como una solución de conectividad de bajo costo que permite ofrecer banda ancha en enlaces de largo alcance lo cual es fundamental para las zonas rurales de la geografía colombiana.

Para predecir el throughput saturado bidimensional se propuso, implementó y validó un modelo "CPS" para el envío de paquetes UDP sobre DCF en redes punto a punto sobre enlaces de largo alcance.

En los enlaces de comunicación en los que se encontraba presente interferencia disminuyó considerablemente el throughput comparado con el valor teórico esperado.

Se encontró asimetria en el flujo de datos cuando se realizaron mediciones del throughput UDP Bidireccional en cada uno de los enlaces punto a punto.

\section{AGRADECIMIENTOS}

Se le reconocen las contribuciones a Ing. C. A. Bravo y al Ing. V. A. Colmenares, Ing. L. M. Meza, Ing. J. D. Moreno, por la contribución y apoyo en la construcción de los prototipos WiFi autónomos y la realización de las pruebas en laboratorio y en campo abierto.

\section{REFERENCIAS}

[1] G. Hernán. Wireless networks and rural development: Opportunities for Latin America. Information technologies and international development, Vol 2, No. 3, pp. 47-56, Spring, Boston: The Massachusetts Institute of technologies, 2005.

[2] C. Luis, Q. River, C. César, L. Liñán. Wild: Wifi based Long Distance. Lima, Pontificia Universidad Católica del Perú, 2009, 180 p.

[3] S, Surana, Designing Sustainable Rural Wireless Networks for Developing Regions, Trabajo de grado (PhD filosofía en ciencia de la computación), Universidad de California, Berkeley ,2009.

[4] A. Gerson, C. Luis, C. David, C. César, E. David, H. Renato, L. Leopoldo, M. Jesús, M. Andrés, M. Eva Juliana, OSUNA Pablo, CHECO Yuri Pa-, PACO Juan, QUIJANDRIA Yvanna, QUISPE River, REY Carlos, SALMERÓN Sandra, SÁNCHEZ Arnau, SANONI, Paola, SEOANE Joaquín, SIMÓ, Javier y VERA Jaime. Redes inalámbricas para zonas rurales, Lima, Pontificia Universidad Católica del Perú Enero 2008, 252 p.

[5] M. Afanasyev, T. Chen, G. M. Voelker, and A. C. Snoeren, "Usage Patterns in an Urban WiFi Network," IEEE/ACM Transactions of Networking, vol. 18, No.5, pp.1359-1372, October 2010.

[6] S. F. Javier, Modelado y Optimización de IEEE802.11 para su Aplicación en el Despliegue de Redes Extensas en Zonas rurales aisladas de Países en Desarrollo. Trabajo de grado (Doctor en ingeniería de telecomunicación). Universidad Politécnica Superior, Escuela superior de ingenieros de telecomunicación, Departamento de Ingeniería y Sistemas Telemáticos 2007.

[7] E. M. María, Guía para el Diseño e implementación de redes inalámbricas en entornos rurales de Perú, Trabajo de grado (Ingeniero de Telecomunicaciones), Escuela Politécnica Superior, Universidad Autónoma de Madrid, 2010.

[8] P. B, Germán, Guía de tecnologías de conectividad para acceso en áreas rurales. Unión internacional de telecomunicaciones, Oficina de desarrollo de las telecomunicaciones, 2007, 84p. 
[9] T, Daniele, G, Alessandro Stefanelli, Riccardo, F. Benedetta, C. Fluvio, Reliability and scalability analysis of Low cost long distance IP-Based wireless networks, innovations for digital inclusion ITU-T Kaleidoscope event, Mar del Plata, 2009.

[10] T. Daniele, G. Alessandro, Stefanelli, Riccardo, F. Benedetta, V. Piergiorgi, Performance of Low Cost Radios in the Implementation of Long Distance Wireless Links, iXem Labs, Politecnico di Torino, Italy, 2008.

[11] T. Daniele, G. Alessandro Stefanelli, Riccardo, F, Benedetta, C. Fluvio, An independent, Low Cost and Open Source Solution for the realization of wireless links over huge multikilometric Distance, p.495-498, IEEE Radio and Wireless Symposium, 2008.

[12] K. P., Rabin. Multi-Tier Network Architecture for Long Distance Rural Wireless Networks in Developing Regions. California, 2009, Trabajo de grado (Ph.D. en fiIosofía en ciencias de las computación). University of California at Berkeley, Electrical Engineering and Computer Science.

[13] S. Anmol, N. Sergiu, P. Rabin, S. Sonesh, BREWER, Eric, S. Lakshminarayanan. Packet Loss Characterization in WiFi-based Long Distance Networks, Universidad de California Berkeley, Universidad de Colorado, Universidad de Nueva York, IEEE INFOCOM, 2007, pp 312-320.

[14] R. A. Andrade, P. H Salas, D. S. Paredes, "Tecnología Wi-Fi”, Argentina, 2008 pp. 1-116.

[15] I. 802.11-2007, Wireless LAN Medium Access Control (MAC) and Physical Layer (PHY) Specifications, Rev. 2007, technical report, IEEE CS, 2007.

[16] Excerpts from FCC Rules part 15 relative to "Unlicensed Spread Spectrum radio systems" believed to be current as of July 23, 1996.

[17] P. Caleb, S. Douglas, G. Dirk, The Stability of The Longley-Rice Irregular Terrain Model for Typical Problems, University of Colorado at Boulder, 2011.

[18] G. A. Hufford, A. G. Longley, W. A. Kissick, "A guide to use of the ITS irregular terrain model in the area prediction mode," U.S. Dep. Commerce, Boulder, CO, NTIA Rep. 82-100, Apr. 1982.

[19] T. S. Rappaport, Wireless Communications Principles and Practice, 2th. Ed., Prentice Hall, New Jersey, pp. 70-71, 2002.

[20] Fabricante de Radios Wi-Fi IEEE802.11 Ubiquity Networks, (online), citado en abril 2011, Disponible en: $<$ http://ubnt.com/>.

[21] F. J. Simo Reigadas, A. Martínez Fernández, F. J. Ramos-López, J. Seoane-Pascual, Modeling and Optimizing IEEE 802.11 DCF for Long-Distance Links", IEEE
Transactions on Mobile Computing, p. 15, Vol. 9, No. 6, 2010.

[22] K. Chebrolu, B. Raman, S. Sen. Long-Distance 802.11b Links: Performance Measurements and Experience. In ACMMOBICOM, 2006.

[23] D . Aguayo, J. Bicket, S. Biswas, G. Judd, and Robert M. Link-level Measurements from an 802.11b Mesh Network. In SIGCOMM, Aug 2004.

[24] P. Barsocchi, G. Oligeri y F. Potorti. Frame error model in rural Wi-Fi networks. IEEE Transactions on wireless communications, Marzo 2009.

[25] P. Guangyu and T. Henderson, Validation of ns-3 802.11b PHY model. Boeing Research and Technology, The Boeing Company, MAY 2009.

[26] M. B. Pursley, Fellow, IEEE, and T. C. Royster IV, Properties and Performance of the IEEE 802.11b Complementary-Code-Key Signal Sets, IEEE TRANSACTIONS ON COMMUNICATIONS, VOL. 57, NO. 2, FEBRUARY 2009.

[27] M. Kwiatkowska, G. Norman and J. Sproston. Probabilistic Model Checking of the IEEE 802.11 Wireless Local Area Network Protocol. In H. Hermanns and R. Segala (editors) Proc. PAPM/PROBMIV'02, volume 2399 of Lecture Notes in Computer Science, pages 169-187, Springer. July 2002.

[28] N. Sandra Salmerón, Parametrización de IEEE 802.11e EDCA para la priorización del tráfico VoIP en redes extensas para zonas rurales de países en vías de desarrollo, Madrid, trabajo de grado Máster, Universidad Rey Juan Carlos, ETSI de Telecomunicación, 2007.

[29] W. Grote, C. Ávila y A. Molina. Análisis de máximo desempeño para un WLAN operando a tasas fijas o adaptativas usando el estándar IEEE802.11a/b/g. Ingeniare. Rev. chil. ing. 2007, Vol.15, No.3, pp. 320-327.

[30] S. Delgadillo, D. Guzmán, A. Muller y W. Grote. Análisis experimental de un ambiente Wi-Fi multicelda. Rev. Fac. Ing. - Univ. Tarapacá [online]. 2005, Vol.13, No.3, pp. 45-52. ISSN 0718-1337.

[31] P. Rabin, N. Sergiu, S. Sonesh, S. Anmol, S. Lakshminarayanan, Eric. Brewer. WiLDNet: Design and Implementation of High Performance WiFi Based Long Distance Networks, TIER Group, Universidad de California Berkeley, Universidad de Colorado, Universidad de Nueva York, Boulder, 2007.

[32 G. Bianchi, "Performance Analysis of the IEEE 802.11 Distributed Coordination Function," IEEE J. Selected Areas in Comm, Vol. 18, No. 3, pp. 535-547, Mar. 2000.

[33] G. Bianchi and I. Tinnirello, "Remarks on IEEE 802.11 DCF Performance Analysis," IEEE Comm. Letters, vol. 9, no. 8, pp. 765-767, Aug. 2005. 
[34] M. Kwiatkowska, G. Norman and J. Sproston. Probabilistic Model Checking of the IEEE 802.11 Wireless Local Area Network Protocol. In H. Hermanns and R. Segala (editors) Proc. PAPM/PROBMIV'02, Vol. 2399 of Lecture Notes in Computer Science, pages 169187, Springer. July 2002.

[35] D. K. Puthal y B. Sahoo, A Finite State Model for IEEE 802.11 Wireless LAN MAC DCF, Emerging Trends in Engineering \& Technology, International Conference on, pp. 258-263, First International Conference on Emerging Trends in Engineering and Technology, 2008.

[36] R. P. Keith, G. Jared,How to Guide on JPerf and IPerf, Wireless LAN profesionals 2011. 